\title{
Digital image watermarking in power system security
}

\author{
Xie Ping ${ }^{1 \text { st }}$,Wang $\mathrm{Xi}^{2 \text { nd }}$,Wang Ying ${ }^{3 \text { rd }}$,Liu Jiehui ${ }^{4 \text { th }}$ \\ School of Control and Computer Engineering Academy \\ North China Electric Power University \\ Beijing, China \\ wx1990linda@126.com
}

\begin{abstract}
In this paper, the digital image watermarking algorithm based on lifting scheme wavelet transform and singular value decomposition (SDV) is proposed and performed on the transmission of the electricity price.It scrambles electricity price to get a set of random watermark signal sequence, uses the ideas about lifting scheme wavelet transform to split, predict and update the information, so as to achieve the image frequency resolution and greatly improve the speed of operation. And it utilizes singular value decomposition algorithm in the watermark embedding and extraction process, which makes invisibility and robustness simultaneously improved.By simulation with Matlab,it shows the algorithm has good validity.
\end{abstract}

Key: digital image watermarking, lifting scheme wavelet transform, singular value decomposition, power system security

\section{INTRODUCTION}

With the popularity of applications of information technology and network technology in power system, the business system in the power industry becomes open, interconnected and standardized, and digital power system has become an inevitable trend. Crossregional connection of power grid has made transactions between regions increasingly frequent, and the safe operation of the power system has also gradually formed a dependence on information systems security. Therefore, promoting research on information security issues for the power industry has gradually become the focus of academia and the business community. However, as an important method of information, digital watermarking technology has good invisibility, robustness and security and can be applied to the information security of power system. This paper studies a kind of digital image watermarking that is based on lifting scheme wavelet transformation and singular value decomposition, and applies it to power system, which not only greatly improves the operation speed and robustness but also effectively ensures the information security in the power system.

\section{IMPROVED DIGITAL WATERMARKING ALGORITHM}

\section{A. Llifting scheme wavelet transformation}

Lifting scheme wavelet transformation is a constructor which is based on time (spatial) domain. It has the characteristic of fast calculation speed and small storage space. To implement the signal frequency decomposition, we split, predict and update the signal on the spatial domain of signal, as is shown in figure 1.

1)Divisions: Split the original signal $\mathrm{s}_{\mathrm{n}}$ into two mutually disjointsubsets of $\left(\mathrm{s}_{\mathrm{n}-1}\right)$ (even sequence) and $\mathrm{O}\left(\mathrm{s}_{\mathrm{n}-1}\right)$ (odd sequence), namely, the Split $\left(s_{n}\right)=\left(E\left(s_{n-1}\right), O\left(s_{n-1}\right)\right) \cdot E\left(s_{n-1}\right)$ and $O\left(s_{n-1}\right)$ are two highly relevant subsets. We can effectively eliminate the correlation of two subsets through improvement steps.

2) Prediction: We can adopt a prediction operator $P$ which has nothing with the data structure in the advantage of correlation among data. Predict $\mathrm{O}\left(\mathrm{s}_{\mathrm{n}-1}\right)$ through subset $\mathrm{E}\left(\mathrm{s}_{\mathrm{n}-1}\right)$, namely, $\mathrm{O}\left(\mathrm{s}_{\mathrm{n}-}\right.$ $\left.{ }_{1}\right)=\mathrm{P}\left(\mathrm{E}\left(\mathrm{s}_{\mathrm{n}-1}\right)\right)$, and use the difference $\mathrm{d}_{\mathrm{n}-1}$ of $\mathrm{O}\left(\mathrm{s}_{\mathrm{n}-1}\right)$ and $\mathrm{P}\left(\mathrm{E}\left(\mathrm{s}_{\mathrm{n}-1}\right)\right)$ as the high frequency coefficient on the first level resolution, namely, $\mathrm{dn}-1=\mathrm{O}\left(\mathrm{s}_{\mathrm{n}-1}\right)-\mathrm{P}\left(\mathrm{E}\left(\mathrm{s}_{\mathrm{n}-1}\right)\right)$.

3) Update: Adopt update operator $\mathrm{U}$ to update the high frequency coefficient $d_{n-1}$, and combine with even sequence to get the corresponding scale coefficient, and then get the low frequency components of signal $s_{n}$, namely, $s_{n-1}=E\left(s_{n-1}\right)+U\left(d_{n-1}\right)$, thereinto $\mathrm{S}_{\mathrm{n}-1}$ is the low frequency coefficients of the next level of resolution.

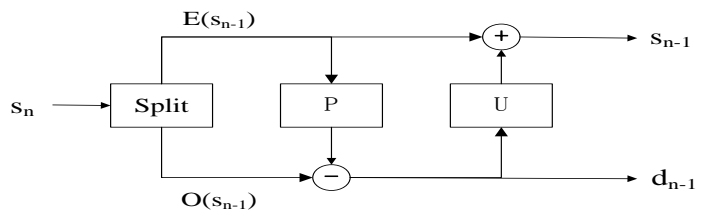

Figure 1 the promotion process

In above three steps, we can decompose the original signals whose resolution is $\mathrm{n}$ into the low frequency signal $\mathrm{s}_{\mathrm{n}-1}$ and high frequency signal $d_{n-1}$ of the next level resolution. For $s_{n-1}$, we can use the same decomposition, and this kind of levels of decomposition is of regularity. Obviously, the process for writing out inverse transformation by wavelet transformation is much easier. Improving method of reconstruction process is shown in figure 2 .

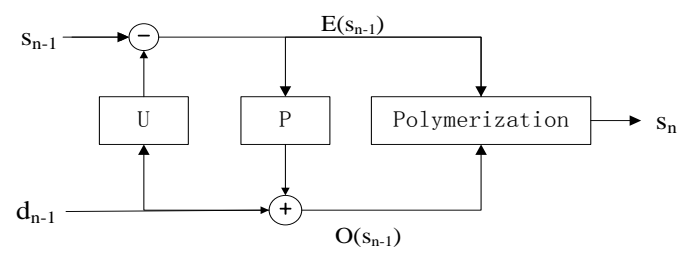

Figure 2 the reconstruction process

\section{B. Three-level wavelet decomposition}

Decompose the original signal by high-pass filter and lowpass filter, and we can get the approximate signal and detail signal which can be called wavelet decomposition. This paper wavelet decomposes images into four subgraph according to twodimensional Mallte tower algorithm of wavelet transform: medium-high frequency detail subgraph of horizontal direction, 
vertical direction and diagonal direction and low frequency approximation subgraph, namely, HH1、HL1、LH1、LL1. Then, decompose LL1 again in the same way and continue to decompose LL2, and finally we can get HH3、 HL3、LH3 and LL3, as is shown in figure 3 . The classification image edge or texture information got after image's subband decomposition concentrate mainly on the greater wavelet coefficient of high frequency subgraph, and the watermarking information carried by diagonal high frequency subband after wavelet decomposition is easy to be damaged. So we can embed watermarking information in intermediate frequency coefficient values to achieve good watermarking performance.

\begin{tabular}{|c|c|c|c|}
\hline LL3 & HL3 & & \\
\cline { 1 - 2 } LH3 & HH3 & HL2 & \multirow{2}{*}{ HL1 } \\
\cline { 1 - 2 } LH2 & HH2 & \\
\cline { 1 - 2 } LH1 & HH1 \\
\hline \multicolumn{2}{|c|}{} & \\
\hline
\end{tabular}

Figure 3 Three-level wavelet decomposition

\section{Singular value decomposition(SVD)}

Digital image is the two-dimensional matrix composed of many nonnegative scalar items. The singular value of image matrix reflects the energy characteristics of image, and the singular vectors reflect the geometric characteristics of the image. The singular value is not sensitive to gray level changes of image, and its subtle changes do not cause the image visual quality decline. If we transpose the image matrix, the singular value won't change. Therefore, the singular value of matrix is of good stability.

The basic principle of method SVD is embedding watermarking into the singular values of image matrix. Specific steps are as follows:

(1) Singular value decompose image matrix $\mathrm{M}$, and get two orthogonal matrix $\mathrm{U}$ and $\mathrm{V}$ and a diagonal matrix $\mathrm{S}$, namely, $\mathrm{M}=\mathrm{USV}^{\mathrm{T}}$;

(2) Read the watermarking image $\mathrm{W}$, and add it to the diagonal matrix $S$, and get the new matrix $S^{\prime}$, namely, $S^{\prime}=$ $\mathrm{S}+\alpha \mathrm{W}$, thereinto, the constant $\alpha>0$ is to adjust the stack strength of the watermarking;

(3) Singular value decompose the new matrix $S$, and get $\mathrm{U}_{1} 、 \mathrm{~S}_{1}$ and $\mathrm{V}_{1}$, namely, $\mathrm{S}^{\prime}=\mathrm{U}_{1} \mathrm{~S}_{1} \mathrm{~V}_{1}{ }^{\mathrm{T}}$;

(4) Multiply matrix $U 、 S_{1}$ and $V^{T}$, and get the watermarking image, $\mathrm{M}^{\prime}=\mathrm{US}_{1} \mathrm{~V}^{\mathrm{T}}$.

\section{APPLICATION OF IMAGE WATERMARKING TECHNOLOGY IN POWER SYSTEM SECURITY}

\section{A. Power system security}

Information security problems in power system are especially important in the Internet era. In "factory net separately, marketing online" mode, electricity market price is an important and sensitive element. Effective protection to price can guarantee the economic benefits of power plant. In order to guarantee electricity market price confidentiality in the transport process and make the buyer and the seller can safely freely, this paper applies digital watermarking technology to the hide of electricity price, makes it difficult to intercept the price information for competitors, and accelerates the transformation speed by using wavelet transform ideas to optimize algorithm, and improves the robustness of the image by using singular value decomposition algorithm.

\section{B. Implement method}

In the case of electricity price information, make a public image in the power system transmission as a carrier, and the implement method has mainly four processes: the pretreatment of watermarking, embedding, extracting and detection.

\section{1) The pretreatment of watermarking}

The pretreatment of watermarking process is to convert into being embedded signal that is fit for embedding into carrier images by original information or other relevant information. Here we make some scrambling transformation for electricity price information and turn it into a set of random watermarking signal W.

\section{2) The embedding of watermarking}

As is shown in figure 4, extract the low and intermediate frequency information from the three-level wavelet decompose transformed watermarking image $\mathrm{W}$, and transform it into binary code flow $\mathrm{W}_{\mathrm{i}}$ as the embedded object. Extract the intermediate frequency coefficient from the three-level wavelet decompose transformed vector image $\mathrm{M}$, and make it as the carrier of embedding watermarking information. Singular value decompose the intermediate frequency coefficient (i.e., a set of low frequency approximation component) and each block of B is used to embed one bit of information. Singular value decompose each block, and get $\mathrm{B}_{\mathrm{i}}=\mathrm{U}_{\mathrm{i}} \mathrm{S}_{\mathrm{i}} \mathrm{V}_{\mathrm{i}}^{\mathrm{T}}$. Then make quantitative processing for the first element $\mu_{\mathrm{i} 1}$ in the diagonal $S_{\mathrm{i}}$ according to the stacking strength $\alpha$ of the given watermarking adjust, and get $\mu_{\mathrm{i} 1}{ }^{\prime}$. Particular way is: (1) if $\mathrm{W}_{\mathrm{i}}=0$, modify the value of the diagonal $\mu_{\mathrm{i} 1}$, and make $\mu_{\mathrm{i} 1}$ equal with the even multiple value of $\alpha$ that is nearest to $\mu_{i 1}$. (2) if $\mathrm{W}_{\mathrm{i}}=1$, modify the value of the diagonal $\mu_{\mathrm{i} 1}$, and make $\mu_{\mathrm{i} 1}$ equal with the odd multiple value of $\alpha$ that is nearest to $\mu_{\mathrm{i} 1}$. Replace the first element $\mu_{\mathrm{i} 1}$ of original diagonal matrix with $\mu_{\mathrm{i} 1}{ }^{\prime}$ and get the new diagonal matrix $\mathrm{S}_{\mathrm{i}}{ }^{\prime}$, and inverse transform according to singular value, namely $\mathrm{B}_{\mathrm{i}}=\mathrm{U}_{\mathrm{i}} \mathrm{S}_{\mathrm{i}}{ }^{\prime} \mathrm{V}_{\mathrm{i}}^{\mathrm{T}}$, and finally get the watermarking image $\mathbf{M}^{\prime}$ after wavelet reconstruction.

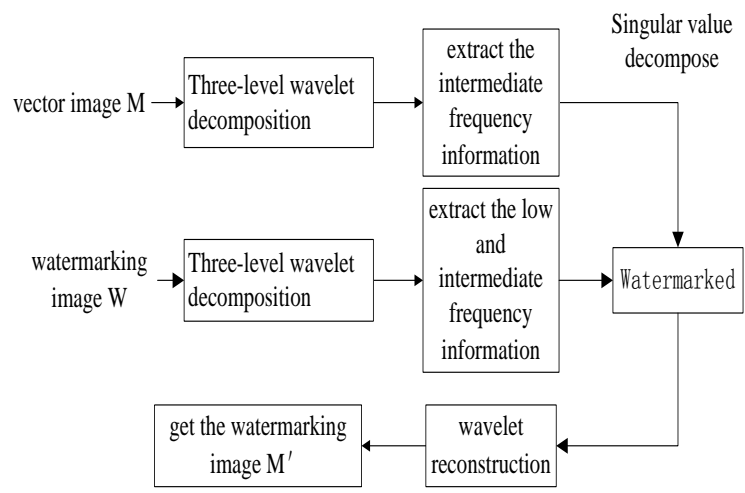

Figure 4 The embedding of watermarking

\section{3 ) The extracting of watermarking}

As is shown in figure 5, extract the low frequency approximation component from the three-level wavelet decompose transformed watermarking image $\mathrm{M}^{\prime}$.Singular value decompose this group of low frequency approximation component, and get $\mathrm{B}_{\mathrm{i}}^{*}=\mathrm{U}_{\mathrm{i}}^{*} \mathrm{~S}_{\mathrm{i}}{ }^{*} \mathrm{~V}_{\mathrm{i}}^{*}$. Then extract watermarking information according to the stacking strength $\alpha$ of the given watermarking adjust. If the diagonal element $\mu_{\mathrm{il}}{ }^{*}$ is near to even multiple of $\alpha$, the extracted data is 0 ; If the diagonal element $\mu_{\mathrm{i} 1}{ }^{*}$ is near to odd multiple of $\alpha$, 
the extracted data is 1 ; Then recover the watermarking information into watermarking image $\mathrm{W}^{\prime}$ after wavelet reconstruction.

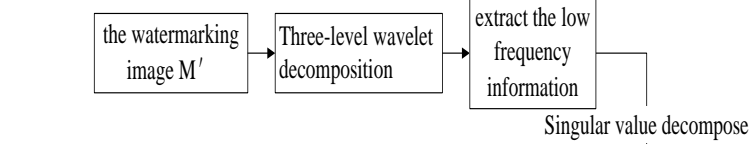

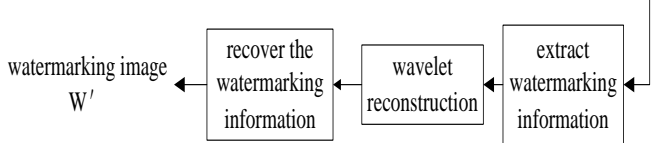

Figure 5 The extracting of watermarking

\section{4) The detection of watermarking}

Measure the robustness of extracted watermarking image $\mathrm{W}^{\prime}$ and original watermarking image $\mathrm{W}$ by normalized correlation coefficient of NC to reflect similarity of original digital watermarking and extracted digital watermarking, as is shown in formula (1).

$$
N C=\frac{\sum_{i} w-n}{\sqrt{\sum_{i} w^{2}} \sqrt{\sum_{i}(w i)^{2}}}
$$

When the value of $\mathrm{NC}$ tends to 1 , it shows that the extracted watermarking and the original watermarking are very close, and it can meet the requirements.

\section{ANALYSIS OF MATLAB SIMULATION RESULT}

Peak signal-to-noise ratio (PSNR) mainly reflects the degree of difference between the watermarking image and the original image. The greater the peak signal-to-noise ratio is ,the better the effect of watermarking image is to the original image. Usually when PSNR $>30$, the difference between the two images is unable to distinguish with the naked eye. so the peak signal-to-noise ratio PSNR is used to measure the concealment of watermarking, and PSNR is shown in formula (2).

$$
P S N R=10 \cdot 1 g \frac{m \times n \max M^{2}}{\sum_{m, n}\left(M-M^{\prime}\right)^{2}}
$$

Thereinto, $m$ and $n$ represent the length and width of image (the number of pixels), and $\mathrm{M}$ represents the original carrier image and $\mathrm{M}^{\prime}$ represents watermarking image.

This experiment adopts the standard image of $512 * 512$ as a carrier image, as is shown in figure 6 , and the Crest image of $64 * 64$ as a being extracted watermarking image, as is shown in figure 7. Use Matlab 7.0 to implement simulation. Here the stacking strength $\alpha$ to adjust watermarking is 0.3 . Figure 8 and figure 9 are watermarking embedded image and extracted watermarking image. As you can see, the difference between it and the original image is not large, so it can satisfy objectively people's visual requirements.

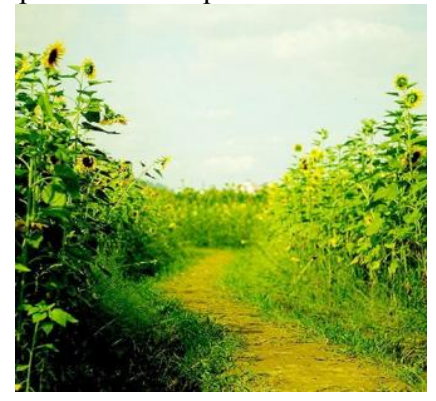

Figure 6 Original image
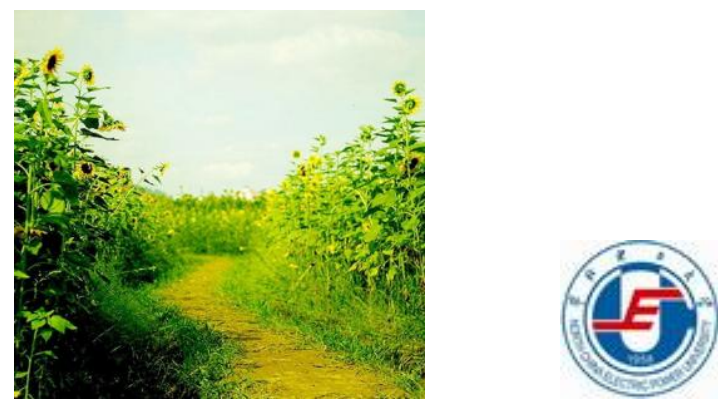

Figure 8 Watermarked image Figure 9 Extracted watermark

Do various image processing and attack operation to watermarking embedded image, analyse the simulation, calculate the peak signal-to-noise ratio, and verify the robustness of the

\begin{tabular}{|c|c|c|}
\hline Type of attack & $N C$ & PSNR \\
\hline No attack & 0.9780 & 39.89 \\
\hline Sharpen & 0.8901 & 30.25 \\
\hline Low pass filter & 0.9754 & 37.54 \\
\hline Wiener filter & 0.9650 & 38.68 \\
\hline Mean filter & 0.9564 & 37.11 \\
\hline Median filter & 0.9645 & 36.32 \\
\hline Salt and pepper noise & 0.7976 & 33.38 \\
\hline JPEG2000 compression & 0.9734 & 38.99 \\
\hline Histogram equalization & 0.8563 & 31.59 \\
\hline
\end{tabular}
algorithm. The experiment result is analysed in table 1 .

Table 1 The experimental results

The experimental results show that the image got from the algorithm after all kinds of processing can still satisfy people's visual requirements, and shows good robustness.

\section{CONCLUSION}

With the interdisciplinary development of electric power industry and the increase the data transmission, information safety of power system appears especially important. As an important method of information hiding, digital watermarking technology is introduced in the power system which can well protect the security of information and achieve the purpose of information hiding. In this paper, application of lifting scheme wavelet transformation and singular value decomposition method makes the realization of the digital watermarking more quickly, strengthens the confidentiality of information, so it is well of robustness.

\section{REFERENCES}

[1] Li Wenwu,Wang Xianpei,Meng Bo.The early study of information security architecture of electric power industry[J] electric power. 2002,35(5):76-79.

[2]Fan Yachun,Zhou Mingquan.Watermark algorithm using lifting-scheme wavelet transform[J]Optics And Precision Engineering. 2009.7

[3]Yu Ruiqing,Li Yongzhong.Research on Digital Watermarking Algorithm Based on Block Wavelet Transform[J]Modern Computer.2007.(12)

[4]Tang Buyao,Li zhiyong,Sun Xingming.Robust Watermarking Algorithm Based on Quantization of Sigular Value[J]Computer Engineering And Applications. 2006(26):63-67.

[5] Liu Ruizhen,Tan Tieniu,SVD Based Digital Watermarking Method[J]Acta Electronica Sinica. 2001, 29(2) 